\title{
Analysis of MFGA to Extract Interesting Rules
}

\author{
Mrinalini Rana \\ Research Scholar, \\ D.A.V Institute of Engineering and Technology \\ Jalandhar, Punjab, India
}

\author{
P S Mann \\ Asstt. Professor, \\ D.A.V Institute of Engineering and Technology \\ Jalandhar, Punjab, India
}

\begin{abstract}
This paper presents a Genetic algorithm based association rule mining in which multi fitness functions are used. Genetic algorithm is used for performing global search. This proposed algorithm generates intersecting association rules from dataset. A fitness function with parameter support is defined for generating frequent itemsets and then other parameters like confidence, lift, leverage etc are used for defining second fitness function for generating association rules. The proposed algorithm is compared with classical Apriori algorithm and also with existing Genetic algorithm for association rule mining on the basis of metrics Support Count, Confidence count, and rule accuracy. Comparisons are also made on different generations.
\end{abstract}

\section{Keywords}

Multi-Fitness Function Genetic algorithm (MFGA), Apriori algorithm, Genetic Algorithm, Crossover Probability, Fitness function, Support , Confidence, Lift, Leverage, Coverage.

\section{INTRODUCTION}

In data mining, Association rule mining becomes one of the important tasks of descriptive technique which can be defined as discovering meaningful patterns from large collection of data. Mining frequent itemset is very fundamental part of association rule mining. Association rule mining is a process of mining or generating interesting rules from the dataset. Market basket analysis can be one of the examples of association rule mining. Generating association rules is generally done by association rule mining algorithm like classical algorithm Apriori, FP-Tree Partition and so on. Instead of using classical Apriori algorithm proposed a Genetic algorithm is used to fine interesting rules.

Genetic algorithm threshold value is its fitness function and in proposed algorithm there are two fitness functions. First fitness function will generate frequent itemsets and second fitness function will generate association rules. Main advantage of Genetic algorithm is that it can perform global search. The analysis was based on proposed algorithm Multi-Fitness function Genetic algorithm (MFGA) based association rule mining. This proposed algorithm generates intersecting rules from dataset. A fitness function is defined for frequent itemset and then different fitness function for generating rules. Second fitness function includes some other interestingness measures than support and confidence to generate relevant rules. The proposed algorithm is compared with classical Apriori algorithm and also with existing Genetic algorithm for association rule mining on the basis of metrics Support Count., Confidence Count, and rule accuracy.

Many algorithms are developed in recent years for mining association rules like Apriori, FP-Tree, Partition algorithm and etc. The subsequent papers [1][8][9] contributed by using multiple objectives like support, confidence, simplicity and etc.
Ashish Ghosh, Bhabesh Nath(2004) discussed: Multiobjective rule mining using genetic algorithms[1], used measures like support count, comprehensibility and interestingness for evaluating interesting rules as their different objectives for mining association rule problem.

Manish Saggar, Ashish Kumar Agrawal, Abhimanyu Lad (2004) discussed: Optimization of Association Rule Mining using Improved Genetic Algorithms [2], main objective is to use genetic algorithm in the discovery of high level prediction rules that perform a global search and perform better with attributes than greedy rule induction algorithms (used in data mining).

Bilal Alata, S. Erhan Akin (2005) discussed: An efficient genetic algorithm for automated mining of both positive and negative quantitative association rules [3], proposed a genetic algorithm as a search strategy for not only positive association rule but also for negative association rules. Proposed algorithm also not relies on minimum support and minimum confidence.

Virendra Kumar Shrivastava, Dr. Parveen Kumar, Dr. K. R. Pardasani(2010) discussed: Extraction of Interesting Association Rules using GA Optimization[4]. In this paper, they used genetic algorithm for extracting association rules. They used measures like support, confidence, interestingness, and completeness.

Amy H.L. Lim a, Chien-Sing Lee a, Murali Raman (2012) discussed: Hybrid genetic algorithm and association rules for mining workflow best practices [5], correlation measure instead of traditional support and confidence in genetic algorithm to driven data dynamically. Correlation fitness function is used to support upward closure in association rule.

Jesmin Nahar et al (2013) discussed: Association rule mining to detect factors which contribute to heart disease in males and females [6], investigates the sick and healthy factors related to heart diseases in males and females by using UCI Cleveland dataset, a biological database. They compared three rule generation algorithms Apriori, Predictive Apriori, Tertius.

Dong Gyu Lee et al (2013) discussed: Discovering Medical Knowledge using Association Rule Mining in Young Adults with Acute Myocardial Infarction[7 proposed association rule mining algorithm that can generate association rules related to hypertension and diabetes from AMI patients having age 45 years old or lesser. They also use measures like lift, leverage and etc

B. Minaei-Bidgoli, R. Barmaki, M. Nasiri(2013) discussed: Mining numerical association rules via multi-objective genetic algorithms[8], proposed a multi-objective genetic algorithm and used measures like confidence, interestingness, and comprehensibility as multiple objectives for genetic algorithm method.

Basheer Mohamad, Al-Maqaleh(2013) discussed: Discovering Interesting Association Rules: A Multi-objective Genetic 
Algorithm Approach[9], proposed a multi-objective genetic algorithm for generating association rules by using measures like support, confidence and simplicity/ comprehensibility.

Bettahally, N. Keshavamurthy, Asad M. Khan, Durga Toshniwal(2013) discussed: Privacy preserving association rule mining over distributed databases using genetic algorithm[10], compared traditional frequent pattern mining algorithm i.e. Apriori algorithm with proposed genetic algorithm in local search.

To overcome the disadvantages of Apriori algorithm, Genetic algorithm can be used for association rule mining. Genetic algorithm uses fitness function as threshold value to evaluate the rules [1] [2] [9] [10]. Above research gap helps to improve Apriori algorithm as well as existing Genetic algorithm which can further be improved.

\section{PROPOSED WORK}

Proposed algorithm is Multi-fitness function Genetic algorithm for association rule mining. This proposed algorithm used two fitness functions for refining the rules. The proposed algorithm is two step algorithms. The first step is aimed to generate rules promptly and then second step is used to refine the rules generated in first step. To refine the rules different interestingness measures are used as fitness function parameters.

\section{MULTI-FITNESS FUNCTION GENETIC ALGORITHM}

Begin:

Step I; Initialize the population;

For each individual; calculate fitness using support as parameter;

For $\mathrm{i}=1$ to maximum generations

[Selection] Select the individuals using Roulette Wheel Selection;

[Crossover] Crossover the parents to form new offspring's;

[Mutation] Mutate new offspring at each locus;

Place new offspring in the new generation;

If fitness of new population> min_supp

Select individuals for next generation;

End If

Next i;

Step II; Initialize the population;

For each individual; Calculate fitness using Confidence, Lift, Leverage, Coverage as parameter;

For $\mathrm{i}=1$ to maximum generations

[Selection] Select the individuals using Roulette Wheel Selection;

[Crossover] Crossover the parents to form new offspring's;

[Mutation] Mutate new offspring at each locus;

Place new offspring in the new generation;

If fitness of new population> min_conf

Select individuals for next generation;

End If

Next i;

End

\section{Operators in proposed algorithm:}

I. Selection Operator: Roulette Wheel selection process is used for selecting the individual's w.r.t fitness function. Roulette Wheel selection process is used for selecting the individual's w.r.t fitness function. procedure for Roulette wheel selection
II. Crossover operator: Single-point crossover is used in proposed algorithm. For example,

100000110101

101010101011

Choose a random bit for crossover and resultant offspring is shown as:

$$
100000101011
$$

101010110101

III. Mutation Operator: This gives a chance that a gene within a chromosome will be flipped means ' 0 ' become ' 1 ' and ' 1 ' become ' 0 '. For example: 100000110101

Choose a random bit and resultant offspring is: 100001110101

\section{Fitness Function:}

The performance of the individual strings is measured by a fitness function. After each iteration, the members are given a performance measure derived from the fitness function, and the "fittest" members of the population will propagate the next iteration.

Fitness Function $=(\mathrm{w} 1 * \operatorname{confidence}(\mathrm{A}->\mathrm{B})+\mathrm{w} 2 *$ coverage $(\mathrm{A}->\mathrm{B})) /(\mathrm{w} 3 * \operatorname{lift}(\mathrm{A}->\mathrm{B})+\mathrm{w} 4 * \operatorname{leverage}(\mathrm{A}->\mathrm{B}))$

where w1 is confidence weight,

$\mathrm{w} 2$ is coverage weight,

w3 is lift weight,

w4 is leverage weight.

For proposed algorithm $w 1=w 3=w 4=1$ and $w 2=2$.

\section{RESULTS}

We present the results we obtained by implementing the algorithms, that have been mentioned in earlier chapters. First we implemented Apriori algorithm to mine significant association rules from a given database. This algorithm first finds out the frequent item sets that have support and confidence values above a pre-specified threshold value. Then it derives the association rules

The experiments were performed on machine Intel ${ }^{\circledR}$ Core 2 Duo CPU $2.00 \mathrm{GHz}$ with $32 \mathrm{bit}$ operating system and software was MatLAB.

Performance Evaluation of Multi-Fitness function Genetic Algorithm:

After getting the Multi-Fitness function Genetic Algorithm for Mushroom database (discussed in previous section), we executed the algorithms for varying number of rules to check their performance and also to have an idea about their relative performances.

We compared the three algorithms (Apriori algorithm, Genetic algorithm, and Multi-Fitness Function Genetic algorithm) on three different measures. Those are mentioned below:

$\begin{array}{ll}\circ & \text { Support Count } \\ \circ & \text { Confidence Count } \\ \bigcirc & \text { Rule Accuracy }\end{array}$

\subsection{Performance analysis with Support Count}

As discussed earlier, the proposed algorithm implements a two stage rule mining. In the first stage simple rule mining is done with fitness function includes support and second stage refines the rules from step1 with fitness function with 
interestingness measures. To analyze the performance of proposed algorithm with Apriori algorithm and existing Genetic Algorithm association rule mining below is the comparison between Apriori algorithm, Genetic algorithm, and Multi-Fitness Function Genetic algorithm based on support count:

Table 1 represents number of rules generated by various support count and Figure 1 represents the graph of comparison between by Apriori algorithm, Genetic algorithm, and Multi-Fitness Function Genetic algorithm.

Table1: Comparison between Apriori algorithm, Genetic algorithm, and Multi-Fitness Function Genetic Algorithm by number of rule generation with varies support count

\begin{tabular}{|c|r|r|c|}
\hline $\begin{array}{l}\text { Support } \\
\text { Count }\end{array}$ & $\begin{array}{l}\text { Apriori } \\
\text { Algorithm }\end{array}$ & $\begin{array}{l}\text { Genetic } \\
\text { Algorithm }\end{array}$ & $\begin{array}{l}\text { Multi-Fitness } \\
\text { FunctionGenetic } \\
\text { Algorithm }\end{array}$ \\
\hline 0.1 & 94698 & 122 & 48 \\
\hline 0.2 & 94689 & 121 & 44 \\
\hline 0.3 & 82602 & 127 & 41 \\
\hline 0.4 & 82540 & 124 & 42 \\
\hline 0.5 & 66368 & 129 & 46 \\
\hline
\end{tabular}

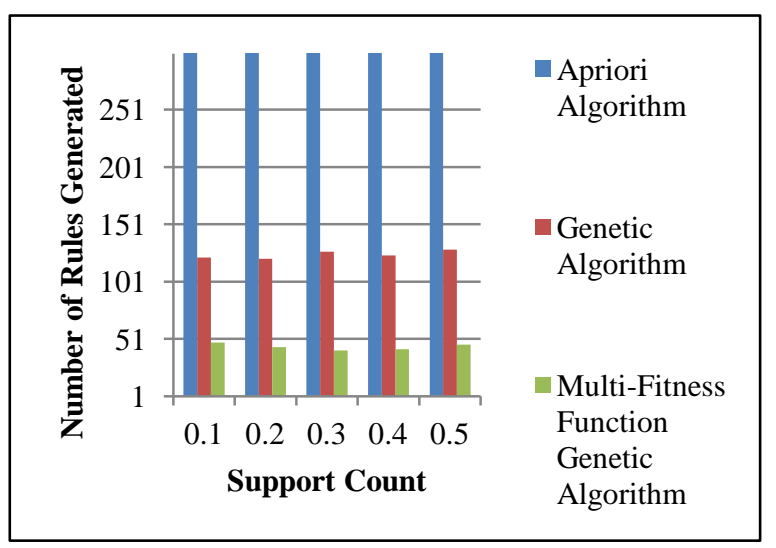

Figure 1: Comparison by various support count

\section{RESULT ANALYSIS ON BASIS ON DIFFERENT MAXIMUM GENERATION:}

Existing genetic algorithm and Multi- Fitness Function Genetic algorithm are evaluated on different generation value varies from 20 to 150 with crossover probability ranges from $0.6-0.9$, as in the proposed algorithm single point crossover is used so crossover probability ranges from $0.6-0.9$. Below are the graphs and the table representing number of rules generated with different generation size.

Below displays rules generated by Apriori algorithm, Genetic algorithm and proposed Multi- Fitness Function Genetic algorithm based on different support count and for the first performance measures number of records were 500
Table2: Existing Genetic Algorithm analysis based on number of Rules generated with different Crossover Probability, Support Count, and Different Maximum Generation

\begin{tabular}{|c|c|c|c|c|c|c|}
\hline \multirow{2}{*}{ 䒠 } & \multirow{2}{*}{ 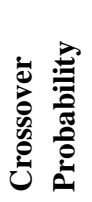 } & \multicolumn{5}{|c|}{ Support Count } \\
\hline & & 0.001 & 0.002 & 0.003 & 0.004 & 0.005 \\
\hline 20 & 0.6 & 140 & 129 & 104 & 112 & 93 \\
\hline 50 & 0.6 & 138 & 129 & 114 & 122 & 83 \\
\hline 100 & 0.6 & 127 & 131 & 106 & 106 & 86 \\
\hline 150 & 0.6 & 133 & 124 & 108 & 111 & 80 \\
\hline 20 & 0.7 & 124 & 134 & 105 & 108 & 83 \\
\hline 50 & 0.7 & 122 & 120 & 121 & 109 & 74 \\
\hline 100 & 0.7 & 123 & 141 & 113 & 104 & 82 \\
\hline 150 & 0.7 & 131 & 138 & 104 & 104 & 82 \\
\hline 20 & 0.8 & 123 & 129 & 108 & 106 & 78 \\
\hline 50 & 0.8 & 123 & 136 & 110 & 107 & 89 \\
\hline 100 & 0.8 & 141 & 119 & 117 & 117 & 85 \\
\hline 150 & 0.8 & 132 & 135 & 109 & 109 & 86 \\
\hline 20 & 0.9 & 130 & 135 & 99 & 116 & 77 \\
\hline 50 & 0.9 & 132 & 132 & 124 & 103 & 83 \\
\hline 100 & 0.9 & 124 & 124 & 106 & 123 & 76 \\
\hline 150 & 0.9 & 123 & 123 & 110 & 101 & 78 \\
\hline
\end{tabular}

Below graphs shows rules generated with crossover probability ranges from $0.6-0.9$ with different generations varies from $20-150$ and the results are of existing genetic algorithm.

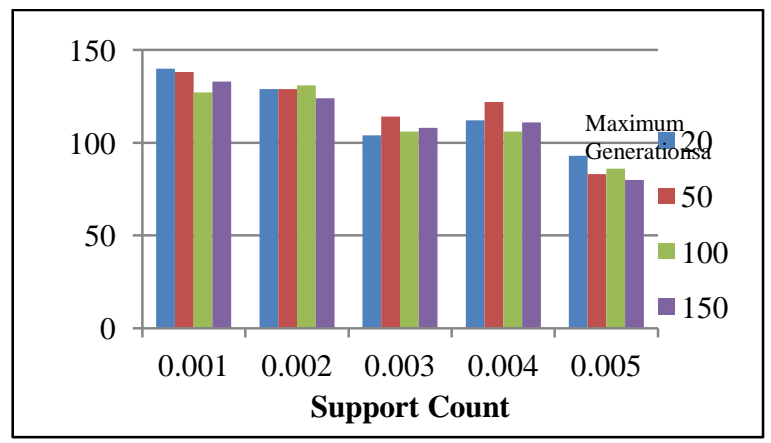

Figure 2: Number of rules generated with Crossover probability 0.6 


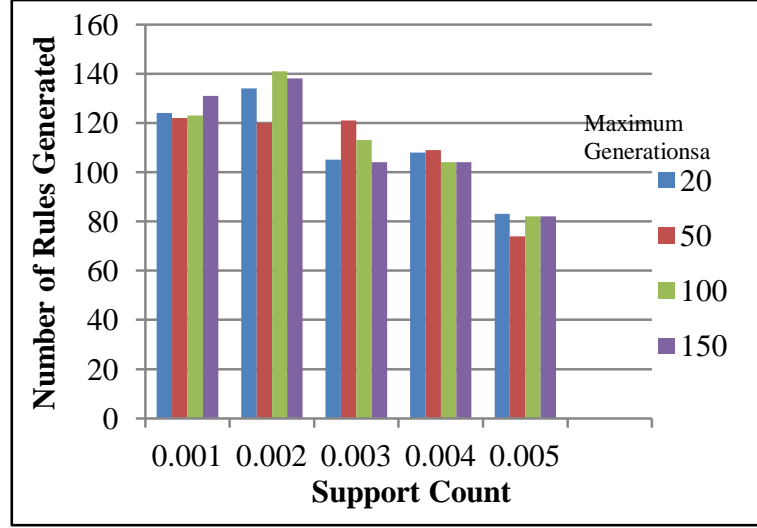

Figure 3 : Number of rules generated with Crossover probability 0.7

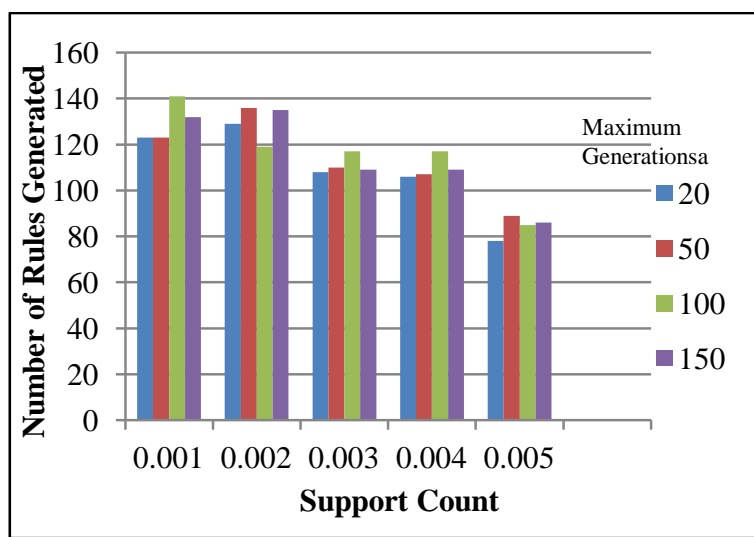

Figure 4: Number of rules generated with Crossover probability 0.8

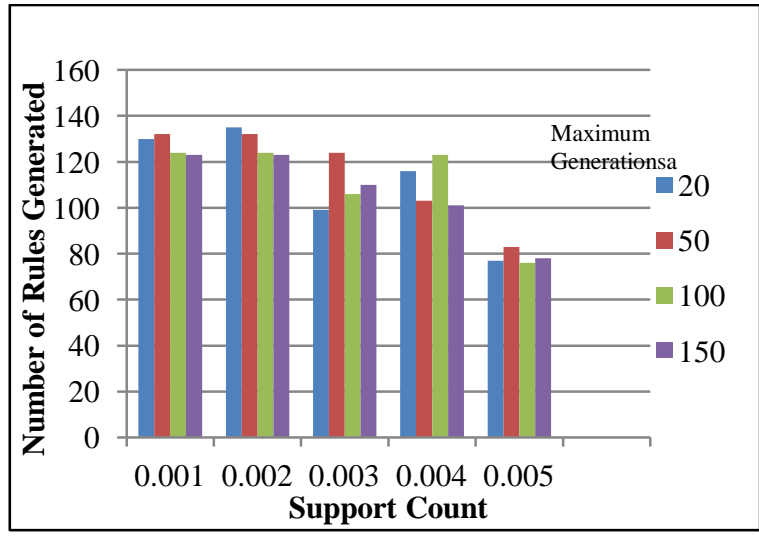

Figure 5: Number of rules generated with Crossover probability 0.9
Below Multi-Fitness function genetic algorithm was evaluated on different generation value varies from 20 to 150 with crossover probability ranges from $0.6-0.9$. Below are the graphs and the table representing number of rules generated with different generation size

Table3: Multi-Fitness Function Genetic Algorithm analysis based on number of Rules generated with different Crossover Probability, Support Count, and Different Maximum Generation

\begin{tabular}{|c|c|c|c|c|c|c|}
\hline \multirow{2}{*}{ 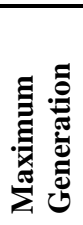 } & \multirow{2}{*}{  } & \multicolumn{5}{|c|}{ Support Count } \\
\hline & & 0.001 & 0.002 & 0.003 & 0.004 & 0.005 \\
\hline 20 & 0.6 & 43 & 43 & 31 & 34 & 28 \\
\hline 50 & 0.6 & 42 & 46 & 35 & 36 & 31 \\
\hline 100 & 0.6 & 47 & 49 & 33 & 35 & 28 \\
\hline 150 & 0.6 & 40 & 47 & 42 & 40 & 27 \\
\hline 20 & 0.7 & 45 & 45 & 41 & 40 & 31 \\
\hline 50 & 0.7 & 44 & 42 & 36 & 40 & 31 \\
\hline 100 & 0.7 & 47 & 44 & 43 & 37 & 24 \\
\hline 150 & 0.7 & 41 & 43 & 33 & 41 & 30 \\
\hline 20 & 0.8 & 40 & 45 & 43 & 37 & 28 \\
\hline 50 & 0.8 & 38 & 46 & 34 & 32 & 31 \\
\hline 100 & 0.8 & 39 & 46 & 36 & 35 & 32 \\
\hline 150 & 0.8 & 47 & 55 & 39 & 39 & 33 \\
\hline 20 & 0.9 & 42 & 47 & 37 & 33 & 28 \\
\hline 50 & 0.9 & 40 & 46 & 35 & 36 & 28 \\
\hline 100 & 0.9 & 39 & 44 & 39 & 38 & 18 \\
\hline 150 & 0.9 & 39 & 46 & 33 & 37 & 22 \\
\hline
\end{tabular}

Above graph and table represents individual analysis of existing Genetic algorithm. Analysis used parameters as Support Count varies from 0.1 to 0.5 . As proposed algorithm uses single point crossover so crossover probability varies from 0.6 to 0.9 but to get accurate results analysis was also conducted on the bases of different crossover probability. Graph (10) showed results with Crossover Probability ranges 0.6-0.9. In this analysis termination condition used was "Maximum Generation", as the loop reached maximum generation (predefined by user) loop terminated and display number of rules generated. So, analysis was also based on different generation (maximum generation) means, generation ranges from 20 to 150 . 


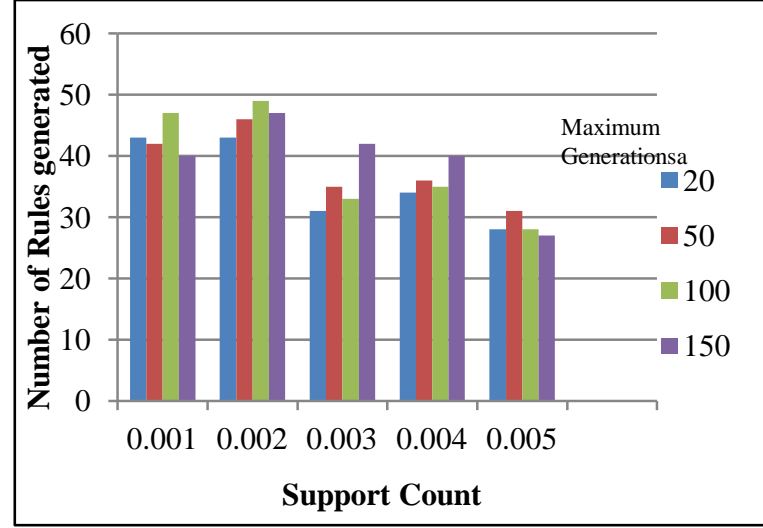

Figure 6: Number of rules generated with Crossover probability 0.6

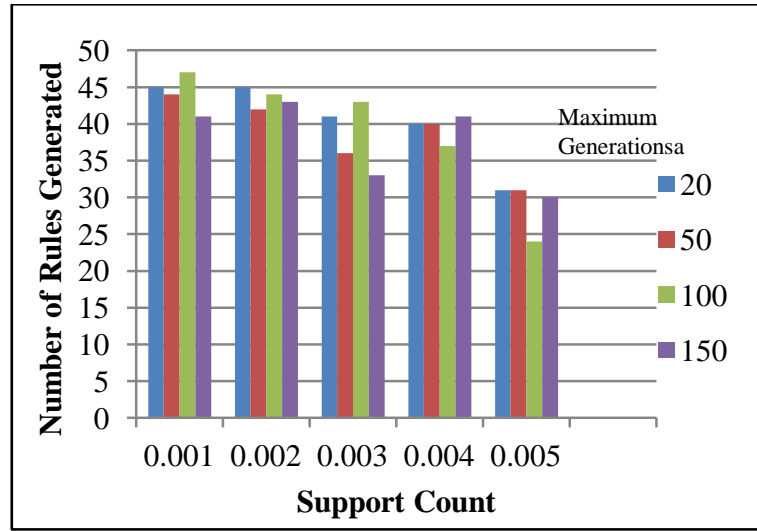

Figure 7: Number of rules generated with Crossover probability 0.7

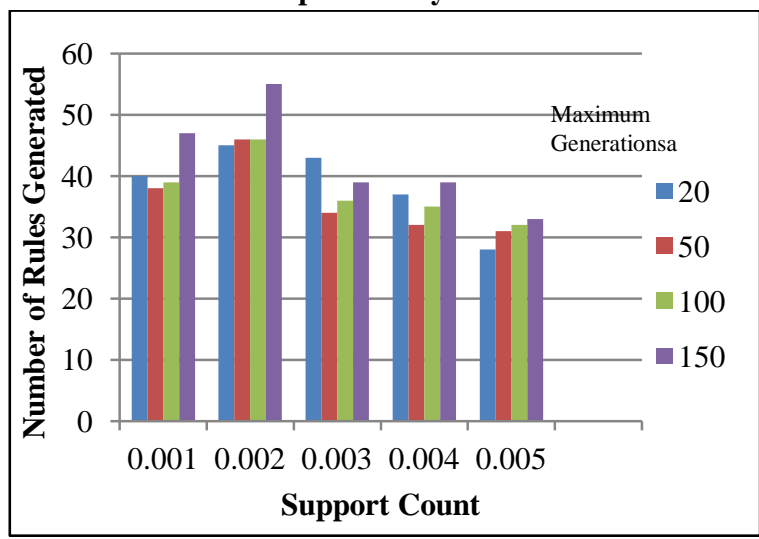

Figure 8: Number of rules generated with Crossover probability 0.8

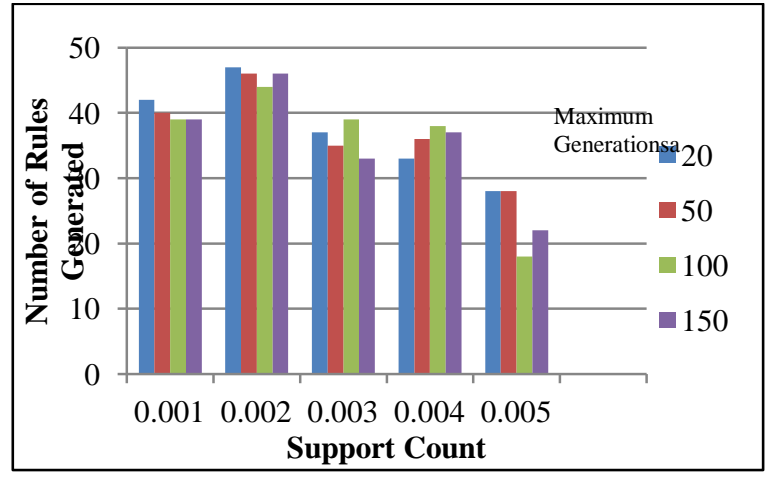

Figure 9: Number of rules generated with Crossover probability 0.9

\subsection{Performance analysis with Confidence count}

Table 6 represents number of rules generated by various confidence counts and Figure 10 represents the graph of comparison between by Apriori algorithm, Genetic algorithm, and Multi-Fitness Function Genetic algorithm.

From above analysis it was observed that for the proposed simulation Crossover probability with 0.8 and maximum generation 100 showed better results so for next two metrics these two parameters were used. Below table includes rules generated with parameters min_support is 0.001, Crossover Probability is 0.8 , maximum generations are 100 with confidence count ranges from 0.4 - 0.6. Below graph depicts the table values graphically.

Table6: Comparison between Apriori algorithm, Genetic algorithm, and Multi-Fitness Function Genetic Algorithm by number of rule generation with varies confidence count

\begin{tabular}{|l|l|l|l|}
\hline $\begin{array}{l}\text { Confidence } \\
\text { Count }\end{array}$ & $\begin{array}{l}\text { Apriori } \\
\text { Algorithm }\end{array}$ & $\begin{array}{l}\text { Genetic } \\
\text { Algorithm }\end{array}$ & $\begin{array}{l}\text { Multi-Fitness } \\
\text { Function Genetic } \\
\text { Algorithm }\end{array}$ \\
\hline 0.4 & 94368 & 129 & 40 \\
\hline 0.45 & 94353 & 133 & 45 \\
\hline 0.5 & 82218 & 127 & 42 \\
\hline 0.55 & 82120 & 137 & 42 \\
\hline 0.6 & 68936 & 119 & 45 \\
\hline
\end{tabular}

Below figure (10) depicts the rules generated by Apriori algorithm, Genetic algorithm, and Multi-Fitness Function Genetic Algorithm graphically. Results shows that proposed algorithm generates better results than other two algorithms with support count equals to 0.001 and number of records were 500 and confidence count varies from 0.4 to 0.6 .

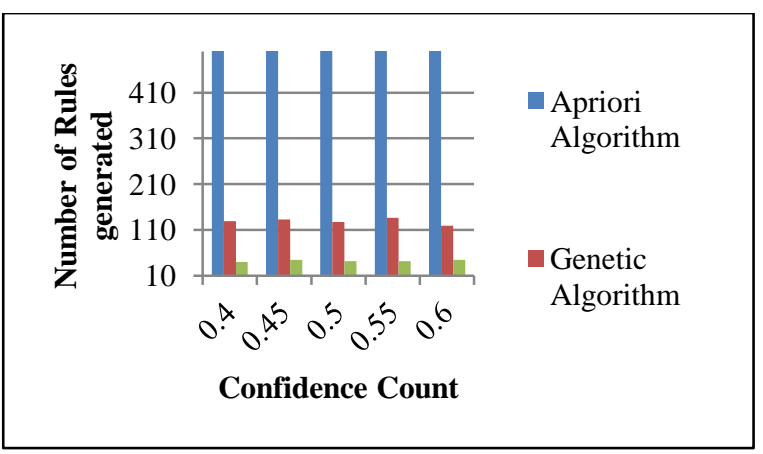

Figure 10: Comparison by various confidence count

\subsection{Performance analysis with metrics Rule Accuracy}

Table 7 represents number of rules generated by metrics rule accuracy and Figure 11 represents the graph of comparison between by Apriori algorithm, Genetic algorithm, and MultiFitness Function Genetic algorithm. Mushroom dataset is divided into 5 records set and then generates the rules and finally rule accuracy concludes that as number of records increases number of rules generation also increases. Number of records varies from 500 to 2500 and with parameters min_support is 0.001, Crossover Probability is 0.8 , maximum generations are 100 with confidence count ranges from 0.4. Below graph depicts the table values graphically. 
Table7: Comparison between Apriori algorithm, Genetic algorithm, and Multi-Fitness Function Genetic Algorithm

by number of rule generation by metrics rule accuracy

\begin{tabular}{|l|l|l|l|}
\hline $\begin{array}{l}\text { Number of } \\
\text { Records }\end{array}$ & $\begin{array}{l}\text { Apriori } \\
\text { Algorithm }\end{array}$ & $\begin{array}{l}\text { Genetic } \\
\text { Algorithm }\end{array}$ & $\begin{array}{l}\text { Multi-Fitness } \\
\text { Function Genetic } \\
\text { Algorithm }\end{array}$ \\
\hline 500 & 94698 & 129 & 40 \\
\hline 1000 & 118392 & 166 & 52 \\
\hline 1500 & 142066 & 235 & 63 \\
\hline 2000 & 164734 & 301 & 71 \\
\hline 2500 & 189314 & 380 & 77 \\
\hline
\end{tabular}

Figure (11) shows result analysis based on Rule accuracy. Mushroom dataset is divided into 5 records set and then generates the rules and finally rule accuracy concludes that as number of records increases number of rules generation also increases

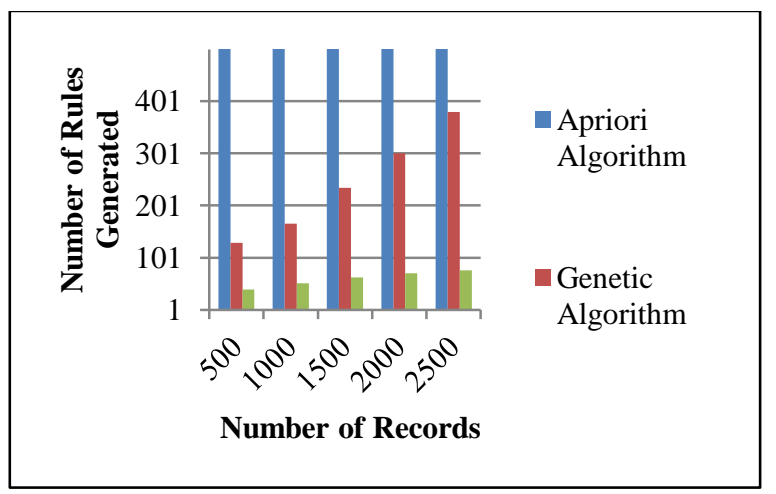

Figure 11: Comparison by Metrics Rule Accuracy

\section{CONCLUSION}

In above simulation work, an effort is made to propose a simple, effective and time saving algorithm for association rule generation in which multi fitness functions can be used as the threshold value for rule evaluation. Some more interestingness measures like lift, leverage, convergence can be used to enhance the fitness function except support and confidence.

The proposed algorithm generates intersecting rules from dataset. A fitness function is defined for frequent itemset and then different fitness function for generating rules. Second fitness function includes some other interestingness measures than support and confidence to generate relevant rules.

Simulation results shows that proposed genetic algorithm generates more efficient and effective rules than other association rule mining techniques such as Apriori and existing genetic algorithm. In the proposed algorithm termination condition involved was maximum generations. Analysis was also taken on the basis of different maximum generation and was also based on different crossover probabilities.

Apriori algorithm generates so many rules because it generates too many frequent itemsets and iteration keep on processing till itemset includes zero items. So to avoid too many relevant and irrelevant rules Apriori algorithm rules were regenerated by existing genetic algorithm and again to optimize those rules proposed algorithm i.e. Multi-Fitness Function Genetic Algorithm was used. Proposed algorithm uses threshold value including parameters of interestingness.
Proposed algorithm can be used for further more applications of data mining. Association Rule Mining based classifiers increases the effectiveness of the rules. Sampling techniques like regression-based sampling or cluster-based sampling will improve the correctness of the rules generated by the algorithm. Genetic algorithm complexity can be reduced by distributed computing. Combination of support, confidence, lift, leverage and conviction can be used to evaluate the interestingness. Genetic algorithm association rule mining can be improved by improving the chromosome representation and fitness function parameters. Distributed computing can also reduced the complexity. In proposed algorithm different termination conditions can be also be used.

\section{ACKNOWLEDGEMENT}

The authors are greatly indebted to the Department of Computer Science and Engineering, D.A.V Institute of Engineering and Technology, Jalandhar for providing excellent lab facilities that make this work possible

\section{REFERENCES}

[1] Ashish Ghosh, Bhabesh Nath," Multi-objective rule mining using genetic algorithms", Information Sciences, Elsevier, Vol.163, pp.123-133, 2004

[2] Manish Saggar, Ashish Kumar Agrawal, Abhimanyu Lad, " Optimization of Association Rule Mining using Improved Genetic Algorithms", IEEE International Conference on Systems, Man and Cybernatics, pp.37253729, 2004.

[3] Bilal Alata, S. Erhan Akin," An efficient genetic algorithm for automated mining of both positive and negative quantitative association rules", Soft Computing, Springer-Verlag, Vol. 10, pp. 230-237, 2005.

[4] Virendra Kumar Shrivastava1 Dr. Parveen Kumar, Dr. K. R. Pardasani, "Extraction of Interesting Association Rules using GA Optimization", Global Journal of Computer Science and Technology, Vol. 10, Issue 5, Ver. 1.0 , pp.81-84, July 2010 .

[5] Amy H.L. Lim a, Chien-Sing Lee a, Murali Raman," Hybrid genetic algorithm and association rules for mining workflow best practices", Expert Systems with Applications, Elsevier, Vol. 39, pp. 10544-10551, 2012.

[6] Jesmin Nahar a, Tasadduq Imama, Kevin S. Tickle a, YiPing Phoebe Chen," Association rule mining to detect factors which contribute to heart disease in males and females ",Expert Systems with Applications, Elsevier, Vol. 40,pp.1086-1093,2013.

[7] Dong Gyu Lee, Kwang Sun Ryu, Mohamed Bashir, Jang-WhanBae, Keun Ho Ryu, " Discovering Medical Knowledge using Association Rule Mining in Young Adults with Acute Myocardial Infarction", Journal of Medical Systems, Springer, Vol.37,Issue 2, 2013.

[8] B. Minaei-Bidgoli, R. Barmaki, M. Nasiri, " Mining numerical association rules via multi-objective genetic algorithms", Information Sciences, Elsevier, 2013.

[9] Basheer Mohamad, Al-Maqaleh, " Discovering Interesting Association Rules: A Multi-objective Genetic Algorithm Approach", International Journal of Applied Information Systems, Vol. 5, No.3, pp.47-52,February 2013.

[10] Bettahally, N. Keshavamurthy, Asad M. Khan ,Durga Toshniwal, "Privacy preserving association rule mining 
over distributed databases using genetic algorithm", Neural Computing and Applications, Springer-Verlag, 2013.

[11] J. Alcalá-Fdez1, A. Fernández1, J. Luengo1, J. Derrac1,S. García2, L. Sánchez3 And F. Herrera1," KEEL Data-Mining Software Tool: Data Set Repository, Integration of Algorithms and Experimental Analysis Framework",J. of Mult.-Valued Logic \& Soft Computing, Vol. 17, pp. 255-287, 2011.

[12] By Jyothi Pillai, O.P.Vyas, "Encapsulation of Soft Computing Approaches within Itemset Mining - A Survey", Global Journal of Computer Science and Technology Software \& Data Engineering, Vol. 12 Issue 15 Versions 1.0, 2012.

[13] Chengfeng Jian, Fang Li, "An Improved Virus Evolutionary Genetic Algorithm For Workflow Mining ", Journal of Theoretical and Applied Information Technology, Vol. 47 No.1, pp.406-411, 2013.

[14] Sanjeev Sharma, Vivek Bahde, Sudhir Sharma, "Optimization of Association Rules Using Genetic Algorithm", International Journal of Soft Computing, pp.75-79, 2007.

[15] Chad Creighton, Samir Hanash, "Mining gene expression databases for association rules", Bioinformatics, Vol. 19 no. 1, pp.79-86, 2003.

[16] Nicolò Flugy, Jesús Alcalá-Fdez, Andrea Bonarini, Francisco Herrera, " Evolutionary Extraction of Association Rules: A Preliminary Study on their Effectiveness", Springer-Verlag, pp. 646-653, 2009.

[17] Ming-Hsiung Ying, Shao-Hsuan Huang, Luen-Ruei Wu, "An Item Selection Strategy Based on Association Rules and Genetic Algorithms", JOURNAL OF SOFTWARE, Vol. 5, No. 12, pp. 1378-1383, 2010.

[18] Rahul Malhotra, Narinder Singh, Yaduvir Singh, "Genetic Algorithms: Concepts, Design for Optimization of Process Controllers", Computer and Information Science, Vol. 4, No. 2, pp.39-54, March 2011.

[19] Wenxiang Dou, Jinglu Hu, Kotaro Hirasawa and Gengfeng Wu, " Quick Response Data Mining Model Using Genetic Algorithm ", SICE Annual Conference, pp. 1214-1219, 2008.

[20] Rupali Haldulakar, Prof. Jitendra Agrawal, "Optimization of Association Rule Mining through Genetic Algorithm", International Journal on Computer Science and Engineering, Vol. 3 No. 3, pp.1252-1259, March 2011.

[21] Soumadip Ghosh, Sushanta Biswas, Debasree Sarkar, Partha Pratim Sarkar, "Mining Frequent Itemsets Using Genetic Algorithm", International Journal of Artificial Intelligence \& Applications, Vol.1, No.4, pp.133-143, October 2010.

[22] Anandhavalli M., Suraj Kumar Sudhanshu, Ayush Kumar, Ghose M.K., "Optimized association rule mining using genetic algorithm", Advances in Information Mining, Vol. 1, Issue 2, pp. 01-04, 2009.

[23] Farah Hanna AL-Zawaidah, Yosef Hasan Jbara, Marwan AL-Abed Abu-Zanona, " An Improved
Algorithm for Mining Association Rules in Large Databases", World of Computer Science and Information Technology Journal, Vol. 1, No. 7, pp.311-316, 2011.

[24] Pradeep Singh Raghav, Nikhil Singh, Satendra Singh Thakur, Priyank Jain, "Optimization Of Multiple Relational Classification Algorithms Using Genetic Algorithm", International Journal of Computer Science and Communication, Vol. 3, No. 1, pp. 215-217, January-June 2012.

[25] D. Kerana Hanirex, Dr. A. Kumaravel, " An Efficient Partition and Two Dimensional Approach For Mining Frequent Itemsets", International Journal of Technological Synthesis and Analysis, Vol. 1 Issue 1, pp. 14-17, December 2012.

[26] Jaishree Singh, Hari Ram, Dr. J.S. Sodhi, "Improving Efficiency of Apriori Algorithm using Transaction Reduction", International Journal of Scientific and Research Publications, Vol. 3, Issue 1, pp. 1-4, January 2013.

[27] Huan $\mathrm{Wu}$, Zhigang Lu, Lin Pan, Rongsheng Xu, "An Improved Apriori-based Algorithm for Association Rules Mining", IEEE Sixth International Conference on Fuzzy Systems and Knowledge Discovery, pp.51-55,2009.

[28] D. Magdalene Delighta Angeline, I. Samuel Peter James, "Association Rule Generation Using Apriori Mend Algorithm for Student's Placement", International Journal of Emerging Sciences, Vol. 2(1), pp. 78-86, March 2012.

[29] R. Uday kiran, P Krishna Reddy, "An Improved Multiple Minimum Support Based Approach to Mine Rare Association Rules", IEEE Symposium on Computational Intelligence and Data Mining, 2009.

[30] Indira K, Kanumani S, "Performance Analysis of Genetic Algorithm for Mining Association Rules",IJCSI, Vol.9, Issue2, No 1 PP.368 - 376, 2012.

[31] Anubha Sharma, Nirupma Tivari, "A Survey of Association Rule Mining Using Genetic Algorithm, International Journal of Computer Applications and Information Technology, Vol.1, Issue1, pp. 5-11, 2012.

[32] Soumadip Ghosh, Sustanta Biswas, Debasree Sarkar, P.P. Sarkar, "Association Rule Mining Algorithm and Genetic Algorithm: A Comparative Study", International Conference on Emerging Applications of Information technology, pp.202-205, 2012.

[33] Wanjun Yu, Xiaochun Wang, Fangyi Wang, Erkang Wang, Bowen Chen, "The Research Of Improved Apriori Algorithm for Mining Association Rules", IEEE International Conference on Communication Technology Proceeding, China, pp. 513- 516, 2008.

[34] Hong GUO, Ya ZHOU, "An Algorithm for Mining Association Rules Based on Improved Genetic Algorithm and its Application", Third International Conference on Genetic and Evolutionary Computing, pp. $117-120,2009$.

[35] K.Y.Fung, C.K Kwong, K.M.W. Siu, K.M. Yu, "A multi-objective genetic algorithm approach to rule mining for affective product design", Expert System with Application, Elsevier, Vol. 39, pp. 7411 - 7419, 2012. 